\title{
Fully Uncoupled Electronically Controllable Sinusoidal Oscillator Employing VD-DIBAs
}

\author{
Data Ram Bhaskar $^{1 *}$, Dinesh Prasad ${ }^{1}$, Kanhaiya Lal Pushkar ${ }^{2}$ \\ ${ }^{1}$ Department of Electronics and Communication Engineering, Faculty of Engineering and Technology, \\ Jamia Millia Islamia, New Delhi, India \\ ${ }^{2}$ Department of Electronics and Communication Engineering, Maharaja Agrasen Institute of Technology, Rohini, \\ New Delhi, India \\ Email: *dbhaskar@jmi.ac.in,dprasad@jmi.ac.in, klpushkar@rediffmail.com
}

Received March 21, 2013; revised April 22, 2013; accepted April 30, 2013

Copyright (C) 2013 Data Ram Bhaskar et al. This is an open access article distributed under the Creative Commons Attribution License, which permits unrestricted use, distribution, and reproduction in any medium, provided the original work is properly cited.

\begin{abstract}
Recently, voltage differencing-differential input buffered amplifiers (VD-DIBA)-based electronically controllable sinusoidal oscillator has been presented that it does not have the capability of complete independence of frequency of oscillation (FO) and condition of oscillation (CO) as well as electronic control of both $\mathrm{CO}$ and FO. In this article, a new fully-uncoupled electronically controllable sinusoidal oscillator using two VD-DIBAs, two grounded capacitors and two resistors has been proposed which offers important advantages such as 1) totally uncoupled and electronically controlled condition of oscillation (CO) and frequency of oscillation (FO); 2) low active and passive sensitivities; and 3) a very good frequency stability factor. The effects of non-idealities of the VD-DIBAs on the proposed oscillator are also investigated. The validity of the proposed formulation has been confirmed by SPICE simulation with TSMC $0.18 \mu \mathrm{m}$ process parameters.
\end{abstract}

Keywords: Sinusoidal Oscillator; Voltage-Mode; VD-DIBA

\section{Introduction}

Sinusoidal oscillators find various applications in signal processing, instrumentation, measurement, communication and control systems. The class of single resistance controlled oscillators (SRCOs) using different active element(s)/device(s) has been of particular interest during the last four decades because of their applications in variable frequency oscillators. However, in these SRCOs, electronic control of $\mathrm{CO}$ and $\mathrm{FO}$ can be obtained by replacing the respective controlling resistor(s) with FET based or CMOS voltage controlled resistor(s). A careful inspection of the available SRCOs reveals that while many oscillators enjoy independent single element control of CO and FO, the class of fully uncoupled oscillators has not been considered adequately in the literature. In fully uncoupled oscillator circuits $\mathrm{CO}$ and FO are determined by two completely different sets of active and/ or passive components, that is none of the active and/or passive components appeared in $\mathrm{CO}$ are involved in $\mathrm{FO}$ and vice versa. This feature is very useful for realizing voltage controlled oscillators as FO can be controlled

\footnotetext{
"Corresponding author.
}

independently without disturbing $\mathrm{CO}$, whereas the flexibility of being able to control $\mathrm{CO}$ independently is advantageous to incorporate amplitude stabilization. In the recent past, number of fully-uncoupled sinusoidal oscillators employing different active element(s)/devices has been introduced see [1-7] and the references cited therein. In references [1-5] the $\mathrm{CO}$ and $\mathrm{FO}$ of the proposed oscillators are adjustable through resistors (the electronic tunability can be established by replacing one of the grounded resistors by JFETs/MOSFETs [8,9]), whereas in case of oscillators presented in references [6,7], both $\mathrm{CO}$ and $\mathrm{FO}$ are electronically controllable. The VDDIBA was introduced by Biolek, Senani, Biolkova and Kolka in [10] since then it has been found to be a useful new active building block in realizing all voltage-mode pass filters [11], inductance simulation [12], universal biquad filter [13] and an electronically controllable sinusoidal oscillator [14]. Although the paper presented by the authors in [14] employs two VD-DIBAs, two grounded capacitors and one grounded resistor but this circuit does not have the capability of complete independence of $\mathrm{CO}$ and FO as well as electronic control (only FO is electronically controllable). Therefore, the purpose of this 
paper is, to propose a new fully uncoupled electronically controllable sinusoidal oscillator employing two VDDIBAs, two grounded capacitors and two resistors, which offers 1) fully uncoupled and electronically controlled $\mathrm{CO}$ and FO, 2) low active and passive sensitivities, and 3) a very good frequency stability factor. The feasibility of the proposed oscillator has been demonstrated by SPICE simulation with TSMC $0.18 \mu \mathrm{m}$ process parameters.

\section{The Proposed Fully Uncoupled Oscillator}

The schematic symbol and behavioral model of the VDDIBA are shown in Figures 1(a) and (b) respectively [11]. The VD-DIBAs can be described by the following set of equations:

$$
\left(\begin{array}{l}
I_{+} \\
I_{-} \\
I_{z} \\
I_{v} \\
V_{w}
\end{array}\right)=\left(\begin{array}{ccccc}
0 & 0 & 0 & 0 & 0 \\
0 & 0 & 0 & 0 & 0 \\
g_{m} & -g_{m} & 0 & 0 & 0 \\
0 & 0 & 0 & 0 & 0 \\
0 & 0 & \pm 1 & \mp 1 & 0
\end{array}\right)\left(\begin{array}{c}
V_{+} \\
V_{-} \\
V_{z} \\
V_{v} \\
I_{w}
\end{array}\right)
$$

The proposed new fully-uncoupled electronically controllable sinusoidal oscillator circuit is shown in Figure 2.

Assuming that the VD-DIBAs are characterized by Equation (1), the characteristic equation (CE) of Figure 2 can be given by:

$$
s^{2}+s \frac{1}{C_{1}}\left(\frac{1}{R_{1}}-g_{m_{1}}\right)+\frac{g_{m_{2}}}{R_{2} C_{1} C_{2}}=0
$$

From this $\mathrm{CE}$, the $\mathrm{CO}$ and $\mathrm{FO}$ can be found as: $\mathrm{CO}$

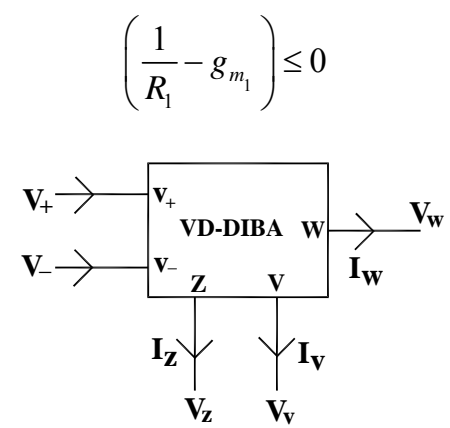

(a)

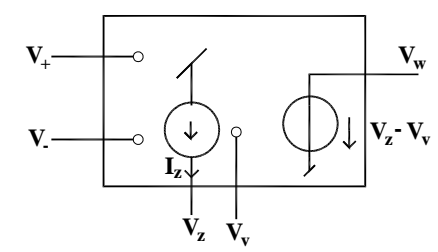

(b)

Figure 1. (a) Schematic symbol; (b) Behavioral model of VD-DIBA.

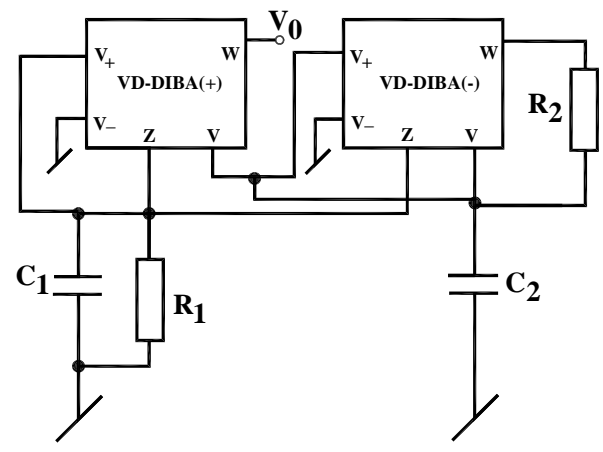

Figure 2. The proposed fully-uncoupled electronically controllable sinusoidal oscillator.

and

FO

$$
\omega_{0}=\sqrt{\frac{g_{m_{2}}}{R_{2} C_{1} C_{2}}}
$$

Therefore, from Equations (3) and (4) it is clear that FO and CO are fully decoupled and electronically controllable i.e. FO is independently controllable by transconductance $g_{m}$ of the VD-DIBA(-), whereas CO is also electronically controllable through the transconductance $g_{m_{1}}$ of VD-DIBA $(+)$.

\section{Non-Ideal Analysis}

Considering the parasitics of VD-DIBA i.e. $R_{Z}$ and $C_{Z}$, the parasitic resistance and the parasitic capacitance of the Z-terminal respectively. Taking the non-idealities into account, namely the voltage of $\mathrm{W}$-terminal $V_{W}=$ $\left( \pm \beta^{+} V_{Z} \mp \beta^{-} V_{V}\right)$ where $\beta^{+}=1-\varepsilon_{p}\left(\varepsilon_{p} \ll 1\right)$ and $\beta^{-}=$ $1-\varepsilon_{n}\left(\varepsilon_{n} \ll 1\right)$ denote the voltage tracking errors of Z-terminal and V-terminal of the VD-DIBA (+/-) respectively, then the expression for $\mathrm{CE}$ becomes:

$$
\begin{aligned}
& s^{2}\left(C_{1}+2 C_{z}\right) C_{2} \\
& +s\left\{C_{2}\left(\frac{1}{R_{1}}-g_{m_{1}}+\frac{2}{R_{z}}\right)+C_{1} \frac{\left(1-\beta_{2}^{-}\right)}{R_{2}}\right. \\
& \left.+C_{z} \frac{2\left(1-\beta_{2}^{-}\right)}{R_{z}}\right\}+\frac{\beta_{2}^{+} g_{m_{2}}}{R_{2}} \\
& +\frac{\left(1-\beta_{2}^{-}\right)}{R_{2}}\left(\frac{1}{R_{1}}-g_{m_{1}}+\frac{2}{R_{z}}\right)=0
\end{aligned}
$$

From Equation (5), the $\mathrm{CO}$ and $\mathrm{FO}$ can be given by: $\mathrm{CO}$ :

$$
\begin{aligned}
& \left\{C_{2}\left(\frac{1}{R_{1}}-g_{m_{1}}\right)\right\}+C_{1} \frac{\left(1-\beta_{2}^{-}\right)}{R_{2}} \\
& +\frac{1}{R_{z}}\left\{2 C_{z}\left(1-\beta_{2}^{-}\right)+2 C_{2}\right\} \leq 0
\end{aligned}
$$


FO:

$$
\omega=\sqrt{\frac{\frac{\beta_{2}^{+} g_{m_{2}}}{R_{2}}+\frac{\left(1-\beta_{2}^{-}\right)}{R_{2}}\left(\frac{1}{R_{1}}-g_{m_{1}}+\frac{2}{R_{z}}\right)}{\left(C_{1}+2 C_{z}\right) C_{2}}}
$$

The sensitivities of $\omega_{0}$ with respect to active and passive elements are calculated as:

$$
\begin{aligned}
& S_{g_{m_{1}}}^{\omega_{0}}=-\frac{\left(1-\beta_{2}^{-}\right) g_{m_{1}}}{2 R_{2}\left\{\frac{\beta_{2}^{+} g_{m_{2}}}{R_{2}}+\frac{\left(1-\beta_{2}^{-}\right)}{R_{2}}\left(\frac{1}{R_{1}}-g_{m_{1}}+\frac{2}{R_{z}}\right)\right\}} \\
& S_{g_{m_{2}}}^{\omega_{0}}=\frac{\beta_{2}^{+} g_{m_{2}}}{2 R_{2}\left\{\frac{\beta_{2}^{+} g_{m_{2}}}{R_{2}}+\frac{\left(1-\beta_{2}^{-}\right)}{R_{2}}\left(\frac{1}{R_{1}}-g_{m_{1}}+\frac{2}{R_{z}}\right)\right\}} \\
& S_{\beta_{2}^{+}}^{\omega_{0}}=\frac{1}{2} \\
& S_{\beta_{2}^{-}}^{\omega_{0}}=-\frac{\beta_{2}^{-}\left(\frac{1}{R_{1}}-g_{m_{1}}+\frac{2}{R_{z}}\right)}{2\left\{\frac{\beta_{2}^{+} g_{m_{2}}}{R_{2}}+\frac{\left(1-\beta_{2}^{-}\right)}{R_{2}}\left(\frac{1}{R_{1}}-g_{m_{1}}+\frac{2}{R_{z}}\right)\right\}} \\
& S_{R_{1}}^{\omega_{0}}=-\frac{\left(1-\beta_{2}^{-}\right)}{2 R_{1} R_{2}\left\{\frac{\beta_{2}^{+} g_{m_{2}}}{R_{2}}+\frac{\left(1-\beta_{2}^{-}\right)}{R_{2}}\left(\frac{1}{R_{1}}-g_{m_{1}}+\frac{2}{R_{z}}\right)\right\}} \\
& S_{R_{2}}^{\omega_{0}}=-\frac{1}{2} \\
& S_{R_{z}}^{\omega_{0}}=-\frac{\left(1-\beta_{2}^{-}\right)}{R_{z} R_{2}\left\{\frac{\beta_{2}^{+} g_{m_{2}}}{R_{2}}+\frac{\left(1-\beta_{2}^{-}\right)}{R_{2}}\left(\frac{1}{R_{1}}-g_{m_{1}}+\frac{2}{R_{z}}\right)\right\}} \\
& S_{C_{1}}^{\omega_{0}}=-\frac{1}{2}=S_{C_{2}}^{\omega_{0}}, S_{C_{z}}^{\omega_{0}}=-1
\end{aligned}
$$

An inspection of Equation (8) reveals that the active and passive sensitivities of $\omega_{0}$ are found to be low.

\section{Frequency Stability}

Frequency stability is an important figure of merit for any sinusoidal oscillator. Using the definition of the frequency stability factor $S^{F}$ as given in $[5,8] S^{F}=$ $\left.\left(\frac{\mathrm{d} \phi(u)}{\mathrm{d} u}\right)\right|_{u=1}$ (where $u=\frac{\omega}{\omega_{0}}$ is the normalized frequency and $\phi(u)$ denotes the phase of the open-loop transfer function), with $C_{1}=C_{2}=C, g_{m_{1}}=\frac{1}{R_{1}}=\frac{1}{R_{2}}=g_{m}$ and $g_{m_{2}}=n g_{m}$, the $S^{F}$ of this oscillator is found to be $2 \sqrt{n}$. Thus the new proposed oscillator circuit offers very high frequency stability factor for larger values of $n$.

\section{Simulation Results}

The proposed sinusoidal oscillator circuit has been simulated using the CMOS-based VD-DIBA [14]. The various component values used were $C_{1}=C_{2}=0.05 \mathrm{nF}, R_{1}=$ $1.67 \mathrm{~K} \Omega$ and $R_{2}=10 \mathrm{~K}$, the CMOS VD-DIBA was biased with $\pm 1 \mathrm{~V}$ D.C. power supplies with $I_{B 1}=I_{B 2}=I_{B 3}=$ $I_{B 4}=I_{B 5}=I_{B 6}=150 \mu \mathrm{A}$ and $I_{B 7}=30 \mu \mathrm{A}$. The transconductances of VD-DIBAs were controlled through the respective bias currents. The SPICE generated output waveforms indicating transient and steady state responses are shown in Figures 3(a) and (b) respectively. From SPICE simulations \{Figures 3(a) and (b) $\}$, the oscillations are observed to be quite stable and the frequency of generated sine wave was found as $731.88 \mathrm{KHz}$. The THD of the output waveform was found as $1.159 \%$. Figure 4 shows the Monte-Carlo simulations which provide the robustness of the oscillator circuit of Figure 2 by taking sample result for $\pm 10 \%$ variations in $R_{1}$. Simulation results, thus, confirm the workability of the pro-

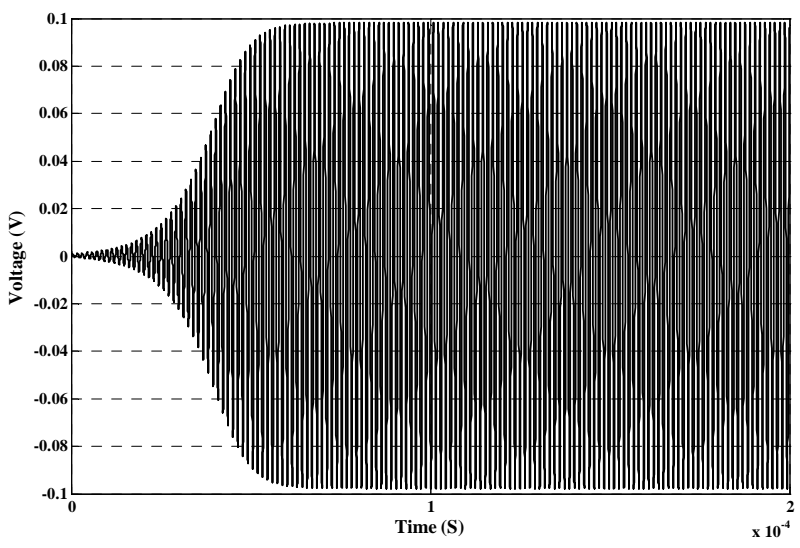

(a)

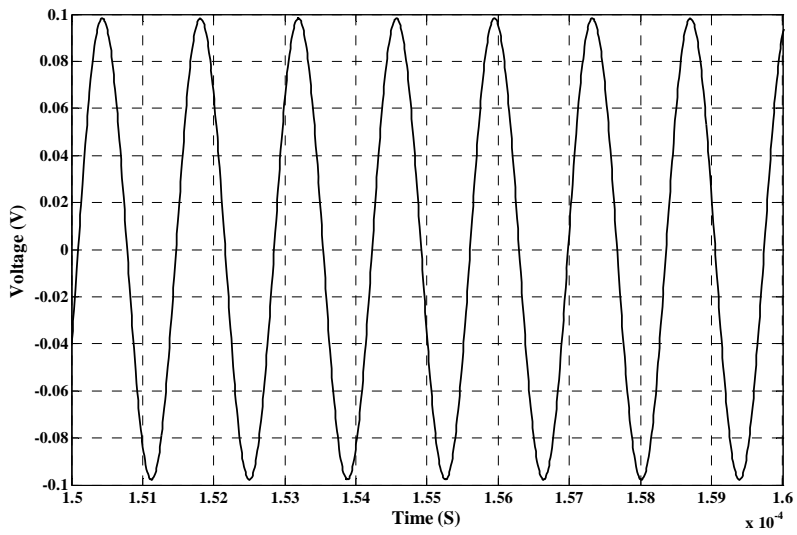

(b)

Figure 3. (a) Transient output waveform; (b) Steady state response of the output. 

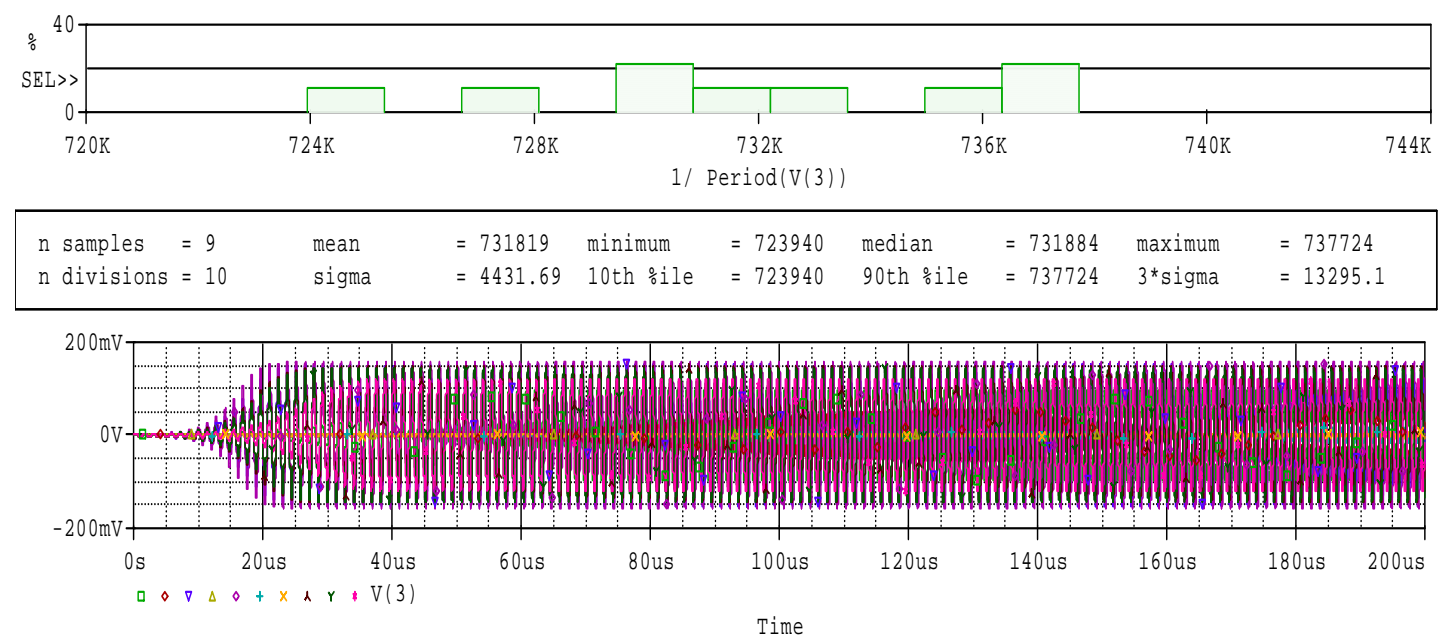

Figure 4. Result of Monte-Carlo simulation of oscillator circuit of Figure 2.

Table 1. Comparison with other previously known fully uncoupled sinusoidal oscillators.

\begin{tabular}{ccccc}
\hline Reference Number & No. of Active Elements & $\begin{array}{c}\text { No. of Passive } \\
\text { Elements }\end{array}$ & $\begin{array}{c}\text { No. of Grounded } \\
\text { Capacitors }\end{array}$ & $\begin{array}{c}\text { Independent Electronic } \\
\text { Tunability in Both CO and FO }\end{array}$ \\
\hline$[1]$ & 2 & 6 & 2 & NO \\
{$[2]$} & 3 & $4-6$ & $2-3$ & NO \\
{$[3]$} & 3 & 5 & 2 & NO \\
{$[4]$} & $1-3$ & $3-7$ & $2-3$ & NO \\
{$[5]$} & 3 & 6 & 2 & YES \\
{$[6]$} & 4 & 2 & 2 & YES \\
{$[7]$} & 2 & 2 & 2 & NO \\
{$[14]$} & 2 & 3 & 2 & YES \\
Proposed & 2 & 4 & 2 & \\
\hline
\end{tabular}

posed oscillator. A comparison with other previously known fully uncoupled sinusoidal oscillators has been given in Table 1.

\section{Concluding Remarks}

A new sinusoidal oscillator with fully decoupled and electronically controllable both frequency of oscillation and condition of oscillation has been presented. The new oscillator configuration also enjoys 1) low active and passive sensitivities and 2) a very good frequency stability factor for larger values of $n$. The robustness of the proposed oscillator circuit has been confirmed by the Monte-Carlo analysis. The workability of the proposed configuration has been established by SPICE simulations with TSMC $0.18 \mu \mathrm{m}$ process parameters.

\section{REFERENCES}

[1] W. Tangsrirat and S. Pisitchalermpong, "CDBA-Based Quadrature Sinusoidal Oscillator," Journal of RF-Engi- neering and Telecommunication (Germany), Vol. 61, No. 3-4, 2007, pp. 102-104.

[2] M. T. Abuelmatti, "New Sinusoidal Oscillators with Fully Uncoupled Control of Oscillation Frequency and Condition Using Three CCII + s," Analog Integrated Circuits and Signal Processing, Vol. 24, No. 3, 2000, pp. 253-261. doi:10.1023/A:1008321911123

[3] D. R. Bhaskar, S. S. Gupta, R. Senani and A. K. Singh, "New CFOA-Based Sinusoidal Oscillators Retaining Independent Control of Oscillation Frequency Even under the Influence of Parasitic Impedances," Analog Integrated Circuits and Signal Processing, Vol. 73, 2012, pp. 427437. doi:10.1007/s10470-012-9896-6

[4] A. M. Soliman, "Current Feedback Operational Amplifier Based Oscillators," Analog Integrated Circuits and Signal Processing, Vol. 23, No. 3, 2000, pp. 45-55. doi:10.1023/A:1008391606459

[5] D. R. Bhaskar, "Realization of Second Order Sinusoidal Oscillator/Filters with Non-Interacting Controls Using CFAs," Journal of RF-Engineering and Telecommunication (Germany), Vol. 57, No. 1-2, 2003, pp. 12-14.

[6] D. R. Bhaskar and R. Senani, "New Linearly Tunable 
CMOS-Compatible OTA-C Oscillators with Non-Interacting Controls," Microelectronics Journal, Vol. 25, No. 2, 1994, pp. 115-123. doi:10.1016/0026-2692(94)90108-2

[7] D. Prasad, M. Srivastava and D. R. Bhaskar, "Electronically Controllable Fully-Uncoupled Explicit CurrentMode Quadrature Oscillator Using VDTAs and Grounded Capacitors," Circuits and Systems, Vol. 4, No. 2, 2013, pp. 169-172. doi:10.4236/cs.2013.42023

[8] D. R. Bhaskar and R. Senani, "New CFOA-Based SingleElement-Controlled Sinusoidal Oscillators," IEEE Transactions on Instrumentation and Measurement (USA), Vol. 55, No. 6, 2006, pp. 2014-2021. doi:10.1109/TIM.2006.884139

[9] S. S. Gupta and R. Senani, "Realisation of Current-Mode SRCOs Using All Grounded Passive Elements," Frequenz, Vol. 57, No. 1-2, 2003, pp. 25-36. doi:10.1515/FREQ.2003.57.1-2.25

[10] D. Biolek, R. Senani, V. Biolkova and Z. Kolka, "Active Elements for Analog Signal Processing; Classification, Review and New Proposals," Radioengineering, Vol. 17,
No. 4, 2008, pp. 15-32.

[11] D. Biolek and V. Biolkova, "First-Order Voltage-Mode All-Pass Filter Employing One Active Element and One Grounded Capacitor," Analog Integrated Circuits and Signal Processing (USA), Vol. 65, No. 1, 2009, pp. 123129. doi:10.1007/s10470-009-9435-2

[12] D. Prasad, D. R. Bhaskar and K. L. Pushkar, "Realization of New Electronically Controllable Grounded and Floating Simulated Inductance Circuits Using Voltage Differencing Differential Input Buffered Amplifiers," Active and Passive Electronic Components, 2011, Article ID 101432.

[13] K. L. Pushkar, D. R. Bhaskar and D. Prasad, "VoltageMode Universal Biquad Filter Employing Single Voltage Differencing Differential Input Buffered Amplifier," Circuits and Systems, Vol. 4, No. 1, 2013, pp. 44-48. doi:10.4236/cs.2013.41008

[14] D. Prasad, D. R. Bhaskar and K. L. Pushkar, "Electronically Controllable Sinusoidal Oscillator Employing CMOS VD-DIBAs," ISRN Electronics, 2013, Article ID 823630. 